\title{
Features for Predicting Absorbable Pulmonary Solid Nodules as Depicted on Thin-Section Computed Tomography
}

\author{
Rui-Yu Lin* \\ Fa-Jin Lv* \\ Bin-Jie Fu $\mathbb{D}$ \\ Wang-Jia Li (D) \\ Zhang-Rui Liang \\ Zhi-Gang Chu (DD
}

Department of Radiology, The First Affiliated Hospital of Chongqing Medical University, Chongqing, People's Republic of China

*These authors contributed equally to this work
Correspondence: Zhi-Gang Chu Department of Radiology, The First Affiliated Hospital of Chongqing Medical University, I\# Youyi Road, Yuanjiagang,

Yuzhong District, Chongqing, 400016,

People's Republic of China

Tel +86 18723032809

Fax +86 2368811487

Email chuzg0815@163.com
Purpose: To investigate the clinical and computed tomography (CT) characteristics of absorbable pulmonary solid nodules (PSNs) and to clarify CT features for distinguishing absorbable PSNs from malignant ones.

Materials and Methods: From January 2015 to February 2021, a total of 316 patients with 348 PSNs (171 absorbable and 177 size-matched malignant) were retrospectively enrolled. Their clinical and CT data were analyzed and compared to determine CT features for predicting absorbable PSNs.

Results: Between absorbable and malignant PSNs, there were significant differences in patients' age, lesions' locations, shapes, homogeneity, borders, distance from the pleura, vacuoles, air bronchograms, lobulation, spiculation, halo sign, multiple concomitant nodules and pleural indentation (each $P<0.05$ ). Multivariate analysis revealed that the independent predictors of absorbable PSNs were the following: patient age $\leq 55$ years (OR, 2.660; 95\% CI, 1.432-4.942; $P=0.002$ ), homogeneous density (OR, 2.487; 95\% CI, 1.107-5.590; $P=$ 0.027), ill-defined border (OR, 5.445; 95\% CI, 1.661-17.846; $P=0.005)$, halo sign (OR, 3.135; 95\% CI, 1.154-8.513; $P=0.025)$, multiple concomitant nodules (OR, 8.700; 95\% CI, 4.401-17.197; $P<0.001$ ), and abutting pleura (OR, 3.759; 95\% CI, 1.407-10.044; $P=0.008$ ). The indicators for malignant PSNs were the following: lobulation (OR, 3.904; 95\% CI, 1.956-7.791; $P<0.001$ ), spiculation (OR, 4.980; 95\% CI, 2.202-11.266, $P<0.001$ ), and pleural indentation (OR, 4.514; 95\% CI, 1.223-16.666; $P=0.024)$.

Conclusion: In patients younger than 55 years, PSNs with homogeneous density, ill-defined border, halo sign, multiple concomitant nodules, and abutting pleura should be highly suspected as absorbable ones.

Keywords: solid nodule, absorbable nodule, follow-up, tomography, x-ray computed

\section{Introduction}

With the widespread application of multislice spiral CT in screening for lung cancer, pulmonary nodules are being detected more frequently. ${ }^{1-3}$ Detected nodules can be divided into solid nodules and subsolid nodules on CT images according to whether or not the lung parenchyma is completely covered by the nodule. ${ }^{4,5}$ Solid nodules are more common than subsolid ones. ${ }^{6}$ Most indeterminate pulmonary solid nodules (PSNs) are benign, and some may be granulomas, infectious lesions, or enlarged lymph nodules, which may decrease in size or be absorbed during follow-up. ${ }^{7}$ Among these, some nodules that could completely disappear during follow-up either spontaneously or during/after antimicrobial therapy were described as absorbable 
PSNs. By contrast, some PSNs are stable or continue growing with various volume doubling times, and these are more likely malignant. ${ }^{8}$

Clinically, management of absorbable and malignant PSNs is quite different. Absorbable PSNs generally do not require special intervention but are easily misdiagnosed as malignancies on first detection. Therefore, it is necessary to distinguish absorbable PSNs from malignant ones. Previous studies have confirmed that PSNs with certain CT manifestations, such as lobulation, spiculation, and pleural indentation, had a high probability of malignancy. $^{9-12}$ There have been several studies of the CT features of absorbable nodules, but most of them have focused on part-solid nodules. ${ }^{5,13-17}$

To date, two studies have focused on absorbable PSNs. ${ }^{7,8}$ Diederich et $\mathrm{al}^{8}$ studied the CT features of resolving nodules with a big portion of PSNs and found that most of them were peripheral, well-defined, and nonlobulated. Zhao et $\mathrm{al}^{7}$ compared the $\mathrm{CT}$ characteristics of resolving and nonresolving PSNs, and found that more resolving PSNs were nonperipheral and spiculated. However, the CT manifestations of absorbable PSNs enrolled in these two studies were not comprehensively evaluated, their results were inconsistent, and there was no comparison between absorbable and malignant PSNs. Thus, it is necessary to further explore the CT features of absorbable PSNs for better understanding them and reducing misdiagnosis. The purpose of this study is to investigate the clinical and CT characteristics of absorbable PSNs and to clarify CT features for distinguishing absorbable PSNs from malignant PSNs.

\section{Patients and Methods}

\section{Patients}

This retrospective study was approved by the institutional review board of the First Affiliated Hospital of Chongqing Medical University, and the requirement for informed consent was waived.

From January 2015 to February 2021, absorbable PSNs and size-matched malignant PSNs were retrospectively collected. The inclusion criteria for absorbable PSNs were as follows: 1) nodules larger than $5 \mathrm{~mm}$ but smaller than $3 \mathrm{~cm}$, and 2) nodules that were completely absorbed during follow-up either spontaneously (118 patients) or during/after antimicrobial therapy (21 patients). The malignant PSNs were all surgically resected and pathologically confirmed. The exclusion criteria for absorbable and malignant PSNs were as follows: 1$)$ absence of thin-section CT $(\leq 1 \mathrm{~mm})$ data and 2) presence of artifacts on CT images affecting evaluation. Finally, a total of 348 PSNs were evaluated, including 171 absorbable and 177 malignant PSNs in 316 patients.

\section{CT Examinations}

Patients were examined using one of the following CT scanners: SOMATOM Perspective (Siemens Healthineers, Erlangen, Germany), SOMATOM Definition Flash (Siemens Healthineers, Erlangen, Germany), or Discovery CT750 HD (GE Healthcare, Milwaukee, WI, USA).

To minimize breathing artifact, all CT scans were performed at the end of inspiration, during a single breathhold. The scan range was from the thoracic entrance to the costophrenic angle. Plain CT scan was acquired with the following settings: tube voltage, $110-130 \mathrm{kVp}$; tube current time, 50-140 mA (using automatic current modulation technology); scanning slice thickness, $5 \mathrm{~mm}$; rotation time, $0.5 \mathrm{~s}$; pitch, 1-1.1; collimation, 0.6 or $0.625 \mathrm{~mm}$; reconstruction slice thickness and interval, 0.625 or $1 \mathrm{~mm}$; matrix, 512×512. All images were reconstructed into 0.625 or $1 \mathrm{~mm}$ slice thickness using a standard algorithm or medium-sharp algorithm.

\section{Image Analysis}

All CT images were reviewed by two senior chest radiologists who were blinded to the clinical data, follow-up CT results, and pathology results. The $\mathrm{CT}$ data were analyzed on a PACS workstation (Carestream Vue PACS) with lung window settings (window level, $-600 \mathrm{HU}$; window width: $1500 \mathrm{HU})$. Any discrepancy between the two radiologists was resolved by consensus.

The following CT features of absorbable and malignant PSNs were analyzed: 1) nodule size (the mean of the longest diameter and the perpendicular diameter on axial CT images), 2) location (upper lobe, middle lobe, or lower lobe), 3) shape (round, oval, or irregular), 4) homogeneity in density (homogeneous or heterogeneous), 5) border (well-defined or ill-defined), (f) distance from the pleura (subpleural, non-subpleural, or abutting pleura), 6) concomitant multiple nodules in other lung fields (yes or no), and 7) other signs (vacuole sign, air bronchogram, lobulation, spiculation, halo sign and pleural indentation). According to the shortest distance between nodule and adjacent pleura, PSNs were divided into non-subpleural ( $>10 \mathrm{~mm})$, subpleural ( $>0 \mathrm{~mm}$ and $\leq 10 \mathrm{~mm})$, and abutting pleura $(0 \mathrm{~mm})$, respectively. 


\section{Statistical Analysis}

The patients' clinical data and CT features of PSNs were statistically analyzed using the SPSS software package (version 22.0, IBM). Continuous variables were expressed as mean \pm standard deviation, and categorical variables were expressed as number and percentage. For comparing differences in variables between absorbable and malignant PSNs, the Mann-Whitney $U$-test was used for patients' ages and nodules sizes, and the Pearson chi-square test was used for gender, smoking history, and frequencies of different CT features. Variables with statistical difference in univariate analysis were further included in logistic regression analysis for determining independent factors for predicting absorbable and malignant PSNs. A $P$-value of $<0.05$ was considered to indicate a statistically significant difference.

\section{Results}

\section{Patients' Clinical Characteristics}

Among the 316 patients, there were 148 men and 168 women, and the mean age was $55.49 \pm 12.25$ years (range, 19-85 years). For 171 absorbable PSNs in 139 patients, the mean follow-up time was $265.88 \pm 270.94$ days (range: 5 1802 days), and the absorption of the PSNs was as follows: $47(27.49 \%)$ within 3 months, $88(51.46 \%)$ within 6 months, $103(60.23 \%)$ within 9 months, 129 (75.44\%) within 12 months, 146 (85.38\%) within 15 months, 154 (90.06\%) within 18 months. The 177 malignant PSNs in 177 patients included $8(4.52 \%)$ adenocarcinoma in situ, 5 (2.82\%) microinvasive adenocarcinoma, 160 (90.40\%) invasive adenocarcinoma, $1(0.56 \%)$ atypical carcinoid, 1 $(0.56 \%)$ small cell lung cancer, $1(0.56 \%)$ alveolar cell carcinoma, and $1(0.56 \%)$ squamous cell carcinoma.

Patients with absorbable PSNs were significantly younger than those with malignant ones $(52.61 \pm 13.78$ years vs $57.75 \pm 10.38$ years, $P=0.001)$. However, there were no significant differences in gender $(P=0.802)$ and smoking history $(P=0.493)$ between these two groups.

\section{CT Characteristics of PSNs}

The CT manifestations of the absorbable and malignant PSNs are summarized in Table 1. There was no significant difference in nodule size between them $(P=0.117)$. Compared with malignant PSNs, absorbable ones were more round or oval, homogeneous, ill-defined, distributed in the lower lobe, abutting pleura, and having halo sign and multiple concomitant nodules (each $P<0.05$ ). By contrast, vacuole sign, air bronchogram, lobulation, spiculation, and
Table I CT Characteristics of Absorbable and Malignant Nodules

\begin{tabular}{|c|c|c|c|}
\hline Characteristics & $\begin{array}{c}\text { Absorbable } \\
\text { PSNs } \\
(n=|7|)\end{array}$ & $\begin{array}{l}\text { Malignant } \\
\text { PSNs } \\
(n=177)\end{array}$ & $P$ value \\
\hline Size $(\mathrm{mm})$ & $9.96 \pm 3.63$ & $10.12 \pm 2.67$ & 0.117 \\
\hline $\begin{array}{l}\text { Multiple concomitant } \\
\text { nodules } \\
\text { No } \\
\text { Yes }\end{array}$ & $\begin{array}{l}66(38.60) \\
105(61.4)\end{array}$ & $\begin{array}{l}149(84.18) \\
28(15.82)\end{array}$ & 0.000 \\
\hline $\begin{array}{l}\text { Lobe location } \\
\text { Upper lobe } \\
\text { Middle lobe } \\
\text { Lower lobe }\end{array}$ & $\begin{array}{c}71(4 I .52) \\
12(7.02) \\
88(51.46)\end{array}$ & $\begin{array}{l}93(52.54) \\
22(12.43) \\
62(35.03)\end{array}$ & 0.039 \\
\hline $\begin{array}{l}\text { Shape } \\
\text { Round/oval } \\
\text { Irregular }\end{array}$ & $\begin{array}{c}164(95.91) \\
7(4.09)\end{array}$ & $\begin{array}{l}153(86.44) \\
24(13.56)\end{array}$ & 0.002 \\
\hline $\begin{array}{l}\text { Homogeneity in } \\
\text { density } \\
\text { Homogeneous } \\
\text { Heterogeneous }\end{array}$ & $\begin{array}{c}\text { I } 38(80.70) \\
33(19.30)\end{array}$ & $\begin{array}{l}107(60.45) \\
70(39.55)\end{array}$ & 0.000 \\
\hline $\begin{array}{l}\text { Border } \\
\text { Well-defined } \\
\text { III-defined }\end{array}$ & $\begin{array}{l}146(85.38) \\
25(14.62)\end{array}$ & $\begin{array}{c}170(96.05) \\
7(3.95)\end{array}$ & 0.001 \\
\hline $\begin{array}{l}\text { Distance to pleura } \\
\text { Abutting pleura } \\
\text { Subpleural/non- } \\
\text { subpleural }\end{array}$ & $\begin{array}{c}44(25.73) \\
127(74.27)\end{array}$ & $\begin{array}{c}8(4.52) \\
169(95.48)\end{array}$ & 0.000 \\
\hline Other signs & & & \\
\hline $\begin{array}{l}\text { Vacuole } \\
\text { Air bronchogram }\end{array}$ & $\begin{array}{l}9(5.26) \\
15(8.77)\end{array}$ & $\begin{array}{l}20(11.30) \\
28(15.82)\end{array}$ & $\begin{array}{l}0.042 \\
0.046\end{array}$ \\
\hline Lobulation & 27 (I5.79) & $92(51.98)$ & 0.000 \\
\hline Spiculation & $14(8.19)$ & $74(4 I .8 I)$ & 0.000 \\
\hline Halo sign & $39(22.8 \mathrm{I})$ & $9(5.08)$ & 0.000 \\
\hline Pleural indentation & $4(2.34)$ & $28(15.82)$ & 0.000 \\
\hline
\end{tabular}

Notes: Except where indicated, data are expressed as number (percentage) or mean \pm standard deviation.

Abbreviation: PSN, pulmonary solid nodules.

pleural indentation were more common in malignant PSNs (each $P<0.05$ ). Moreover, for PSNs abutting pleura, most of the absorbable ones had a broad basement (34, 77.27\%), while acute angles between the malignant PSNs and pleura were usually observed $(7,87.50 \%)$.

\section{Multiple Analysis for Absorbable and Malignant PSNs}

The independent factors for distinguishing absorbable and malignant PSNs revealed by logistic regression are 
summarized in Table 2. Patients younger than 55 years, PSNs with homogeneous density, ill-defined border, halo sign, multiple concomitant nodules, and abutting pleura were independent factors for predicting absorbable PSNs $(P<0.05)$ (Figure 1), while lobulation, spiculation, and pleural indentation were shown to be significantly associated with malignant PSNs $(P<0.05)$ (Figure 2).

\section{Discussion}

It is well known that a substantial proportion of pulmonary nodules is found in lung cancer screening is benign, mostly caused by inflammation or hemorrhage, and usually disappears during follow-up. ${ }^{8,18}$ In clinical practice, some absorbable PSNs may be misdiagnosed as malignancies, which require markedly different clinical management. Therefore,

Table 2 Multivariate Logistic Regression for Predicting Absorbable PSNs

\begin{tabular}{|c|c|c|}
\hline Variable & Odds Ratio (95\% Cl) & $P$ value \\
\hline Age & & 0.002 \\
\hline$>55$ years & I & \\
\hline$\leq 55$ years & $2.660(1.432,4.942)$ & \\
\hline Multiple concomitant nodules & & 0.000 \\
\hline No & 1 & \\
\hline Yes & $8.700(4.401,17.197)$ & \\
\hline Homogeneity in density & & 0.027 \\
\hline Heterogeneous & 1 & \\
\hline Homogeneous & $2.487(1.107,5.590)$ & \\
\hline Border & & 0.005 \\
\hline Well-defined & I & \\
\hline III-defined & $5.445(1.661,17.846)$ & \\
\hline Distance to pleura & & 0.008 \\
\hline Subpleural/ non-subpleural & 1 & \\
\hline Abutting to pleura & $3.759(1.407,10.044)$ & \\
\hline Lobulation & & 0.000 \\
\hline No & $3.904(1.956,7.791)$ & \\
\hline Yes & I & \\
\hline Spiculation & & 0.000 \\
\hline No & $4.980(2.202,11.266)$ & \\
\hline Yes & 1 & \\
\hline Halo sign & & 0.025 \\
\hline No & I & \\
\hline Yes & $3.135(1.154,8.513)$ & \\
\hline Pleural indentation & & 0.024 \\
\hline No & $4.514(1.223,16.666)$ & \\
\hline Yes & 1 & \\
\hline
\end{tabular}

understanding the differences between absorbable and malignant PSNs is very important. The present study found that absorbable PSNs were usually detected in patients younger than 55 years, with homogeneous density, ill-defined border, halo sign, multiple concomitant nodules, and abutting pleura. By contrast, malignant PSNs usually had lobulation, spiculation and pleural indentation.

It has been reported that the incidence of malignant pulmonary nodules increases with age as immunity decreases and total exposure to carcinogens increases. ${ }^{19,20}$ In this study, patients younger ( $\leq 55$ years) was an independent predictor of absorbable PSNs, consistent with earlier findings. ${ }^{14}$ However, there was no significant difference for gender and smoking history between patients with absorbable and malignant PSNs. Thus, only age may be an important clinical factor in determining whether nodules are absorbable or malignant.

Owing to the growth mode of malignant tumors, retained airspace, dilated bronchioles, and the presence of vacuoles make malignant PSNs more likely to be heterogeneous. ${ }^{21}$ In contrast, most absorbable PSNs are caused by inflammation, accumulating of inflammatory cells and exudation in the central zone, and spread to the surrounding lung tissue, making them homogeneous but ill-defined. In this study, more absorbable PSNs than malignant ones had homogeneous density, illdefined border, and halo sign, which were independent predictors. However, the incidence of latter two features was relatively infrequent, which may reflect different stages of inflammation, corresponding to different CT features. A previous study showed that a higher percentage (23\%) of resolving nodules were ill-defined, ${ }^{8}$ and this may be due to its inclusion of some part-solid nodules. Thus, a homogeneous PSN with ill-defined border or halo sign should first be suspected as a benign lesion.

Lobulation, spiculation, and pleural indentation were more likely to be detected in malignant pulmonary tumors because of the different growth rate in different directions, obstruction of surrounding structures, tumor cells invading peripheral tissues, and scar contraction. ${ }^{10-12}$ The current study also found that lobulation, spiculation, and pleural indentation more frequently occurred in malignant PSNs. However, Zhao et $\mathrm{al}^{7}$ found that the incidence of lobulation was not significantly different between resolving and nonresolving PSNs, and that spiculation was more common in resolving PSNs. These discrepancies with our findings may reflect the different sampling methodologies used. In their study, the malignant PSNs accounted for only $3.11 \%$ of nonresolving nodules. Therefore, we believe that lobulation, spiculation, and pleural indentation 


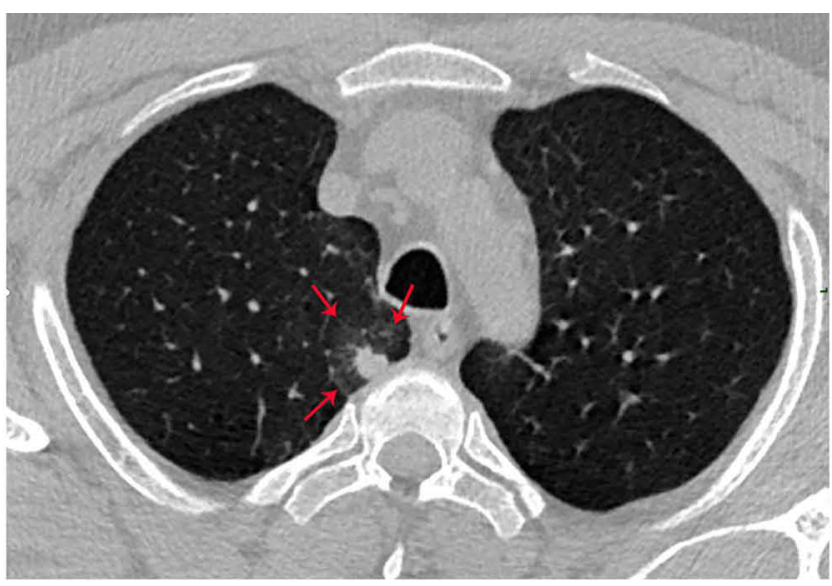

A

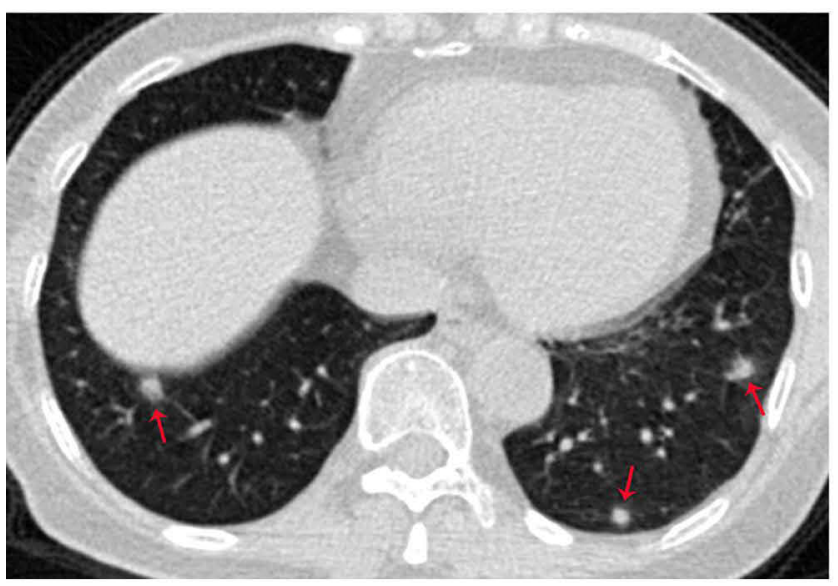

C

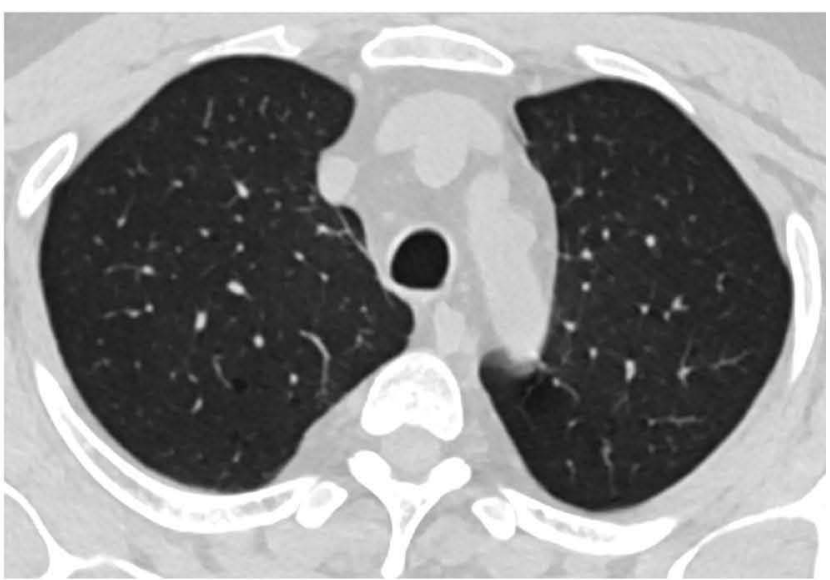

B

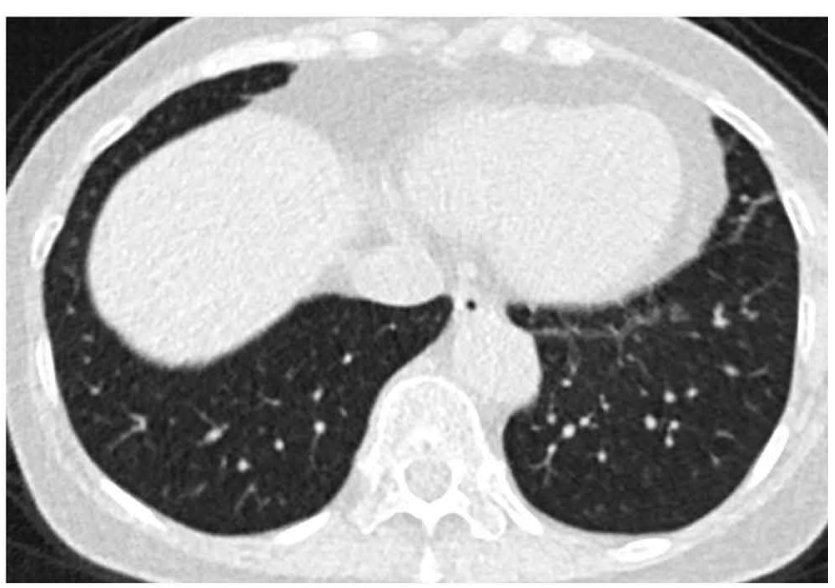

D

Figure I Absorbable PSNs on CT images. (A) Axial CT image in a 50-year-old male shows a round, homogeneous, and well-defined PSN that has a halo sign (arrows) and abuts pleura with a broad basement located in the right upper lobe. (B) It had disappeared at I-month follow-up. (C) Axial CT image in a 65-year-old female shows multiple, round or oval, ill-defined PSNs (arrow) located in the bilateral lower lobes. (D) They had disappeared at 10-month follow-up.

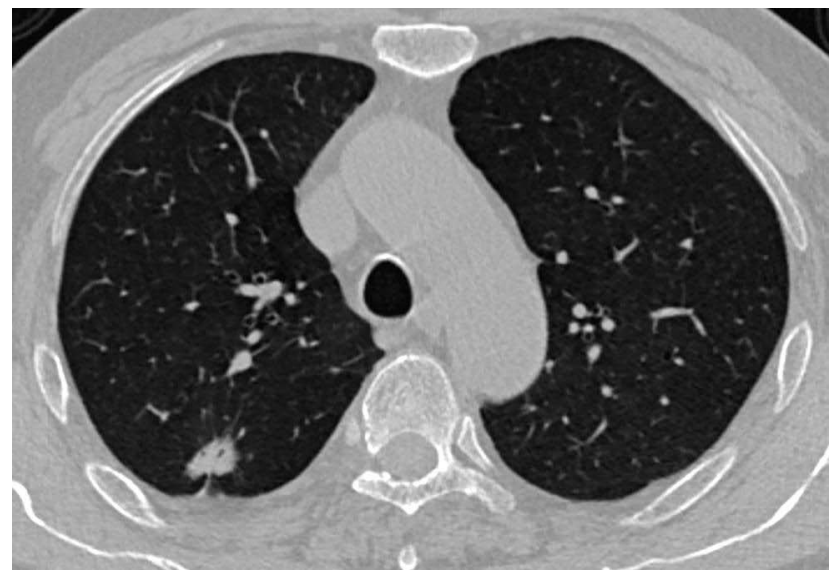

Figure 2 Malignant PSN on CT image. Axial CT image in a 76-year-old male shows a lobulated, heterogeneous, and well-defined PSN with air bronchogram, spiculation, and pleural indentation located in the right upper lobe. It was confirmed as invasive adenocarcinoma after surgical resection. are useful for distinguishing absorbable PSNs from malignant ones, and their value for discriminating between malignant and benign PSNs should be further verified.

Zhao et $\mathrm{al}^{7}$ reported that nonperipheral PSNs were more likely to be absorbable than peripheral ones. However, this study showed that absorbable PSNs were more prone to abutting pleura than malignant ones and that $77.27 \%$ of them had a broad basement, which was consistent with the $\mathrm{CT}$ features of inflammatory lesions. ${ }^{22}$ Inflammation frequently involves subpleural lung tissues because of abundant alveoli. The ease of spread into distal tissue may account for these differences. The present study discovered that multiple concomitant nodules in other lung fields was an important predictor of absorbable PSNs, similar to previous findings. ${ }^{10,16}$ Therefore, when an indetermined PSNs is found, concomitant nodules in other lung fields should be evaluated. 
There are several limitations in our study. First, histological evidence could not be obtained for absorbable nodules, and their pathological basis could only be speculated based on CT features. Second, the follow-up period of patients with absorbable PSNs was irregular in this retrospective study, so the exact absorption time of nodules could not be determined. Zhao et $\mathrm{al}^{7}$ reported that more than $75 \%$ of resolving PSNs nodules disappeared within 3 months. In this study, 51\%, 75\%, and $90 \%$ of absorbable PSNs disappeared within 6,12 , and 18 months, respectively. Thus, follow-up after initial CT examination could exclude a considerable number of benign lesions. It is recommended that the follow-up intervals for patients with suspected absorbable PSNs should be $3,6,12$, and 18 months based on their changes. Third, this study only included PSNs lager than $5 \mathrm{~mm}$ because those less than $5 \mathrm{~mm}$ were numerous and the preferred treatment for them was follow-up.

In conclusion, absorbable PSNs showed different CT features from malignant ones. Homogeneous density, illdefined border, halo sign, multiple concomitant nodules, and abutting pleura have great value in determining whether PSNs are possibly absorbable. In contrast, lobulation, spiculation, and pleural indentation frequently indicate malignant PSNs. Understanding the differences in CT features between absorbable and malignant PSNs will be helpful in differentiating nodules and directing subsequent treatment, avoiding unnecessary surgical resection.

\section{Ethics Statement}

The study was conducted in accordance with the Declaration of Helsinki, and the protocol was approved by the Ethics Committee of the First Affiliated Hospital of Chongqing Medical University (No. 2019-062), which absolved the need for written informed consent because of the retrospective study. All personal identification data were anonymized and de-identified before analysis.

\section{Funding}

This study was supported by the National Natural Science Foundation of China (81601545) and Chongqing Health and Family Planning Commission Foundation (2016MSXM018) of China.

\section{Disclosure}

All authors declare no conflicts of interest for this work.

\section{References}

1. Hasegawa M, Sone S, Takashima S, et al. Growth rate of small lung cancers detected on mass CT screening. Br $J$ Radiol. 2000;73:1252-1259. doi:10.1259/bjr.73.876.11205667

2. Heuvelmans MA, Walter JE, Peters RB, et al. Relationship between nodule count and lung cancer probability in baseline $\mathrm{CT}$ lung cancer screening: the NELSON study. Lung Cancer. 2017;113:45-50. doi:10.1016/j.lungcan.2017.08.023

3. Walter JE, Heuvelmans MA, de Jong PA, et al. Occurrence and lung cancer probability of new solid nodules at incidence screening with low-dose CT: analysis of data from the randomised, controlled NELSON trial. Lancet Oncol. 2016;17:907-916. doi:10.1016/ S1470-2045(16)30069-9

4. Henschke CI, Yankelevitz DF, Mirtcheva R, et al. CT screening for lung cancer: frequency and significance of part-solid and nonsolid nodules. AJR Am J Roentgenol. 2002;178:1053-1057. doi:10.2214/ ajr.178.5.1781053

5. Choi WS, Park CM, Song YS, Lee SM, Wi JY, Goo JM. Transient subsolid nodules in patients with extrapulmonary malignancies: their frequency and differential features. Acta Radiol. 2015;56:428-437. doi: $10.1177 / 0284185114528325$

6. Veronesi G, Bellomi M, Mulshine JL, et al. Lung cancer screening with low-dose computed tomography: a non-invasive diagnostic protocol for baseline lung nodules. Lung Cancer. 2008;61:340-349. doi:10.1016/j.lungcan.2008.01.001

7. Zhao YR, Heuvelmans MA, Dorrius MD, et al. Features of resolving and nonresolving indeterminate pulmonary nodules at follow-up CT: the NELSON study. Radiology. 2014;270:872-879. doi:10.1148/ radiol.13130332

8. Diederich S, Hansen J, Wormanns D. Resolving small pulmonary nodules: CT features. Eur Radiol. 2005;15:2064-2069. doi:10.1007/ s00330-005-2836-4

9. Xu DM, van Klaveren RJ, de Bock GH, et al. Limited value of shape, margin and CT density in the discrimination between benign and malignant screen detected solid pulmonary nodules of the NELSON trial. Eur $J$ Radiol. 2008;68:347-352. doi:10.1016/j. ejrad.2007.08.027

10. Lee SM, Park CM, Goo JM, Lee HJ, Wi JY, Kang CH. Invasive pulmonary adenocarcinomas versus preinvasive lesions appearing as ground-glass nodules: differentiation by using CT features. Radiology. 2013;268:265-273. doi:10.1148/radiol.13120949

11. Lee HJ, Goo JM, Lee CH, et al. Predictive CT findings of malignancy in ground-glass nodules on thin-section chest $\mathrm{CT}$ : the effects on radiologist performance. Eur Radiol. 2009;19:552-560. doi:10.1007/s00330-008-1188-2

12. Oh JY, Kwon SY, Yoon HI, et al. Clinical significance of a solitary ground-glass opacity (GGO) lesion of the lung detected by chest CT. Lung Cancer. 2007;55:67-73. doi:10.1016/j.lungcan.2006.09.009

13. Qi L, Xue K, Li C, et al. Analysis of CT morphologic features and attenuation for differentiating among transient lesions, atypical adenomatous hyperplasia, adenocarcinoma in situ, minimally invasive and invasive adenocarcinoma presenting as pure ground-glass nodules. Sci Rep. 2019;9:14586. doi:10.1038/s41598-019-50989-1

14. Yu JY, Lee B, Ju S, et al. Proportion and characteristics of transient nodules in a retrospective analysis of pulmonary nodules. Thorac Cancer. 2012;3:224-228. doi:10.1111/j.1759-7714.2011.00101.x

15. Lee SM, Park CM, Goo JM, et al. Transient part-solid nodules detected at screening thin-section CT for lung cancer: comparison with persistent part-solid nodules. Radiology. 2010;255:242-251. doi:10.1148/radiol.09090547

16. Chung K, Ciompi F, Scholten ET, et al. Visual discrimination of screen-detected persistent from transient subsolid nodules: an observer study. PLoS One. 2018;13:e191874. doi:10.1371/journal. pone. 0191874 
17. Felix L, Serra-Tosio G, Lantuejoul S, et al. CT characteristics of resolving ground-glass opacities in a lung cancer screening programme. Eur J Radiol. 2011;77:410-416. doi:10.1016/j.ejrad.2009.09.008

18. Bankoff MS, McEniff NJ, Bhadelia RA, Garcia-Moliner M, Daly BD. Prevalence of pathologically proven intrapulmonary lymph nodes and their appearance on CT. AJR Am J Roentgenol. 1996;167:629-630. doi:10.2214/ajr.167.3.8751667

19. Chu ZG, Li WJ, Fu BJ, et al. CT characteristics for predicting invasiveness in pulmonary pure ground-glass nodules. AJR Am J Roentgenol. 2020;215:351-358. doi:10.2214/AJR.19.22381

20. Cui X, Han D, Heuvelmans MA, et al. Clinical characteristics and work-up of small to intermediate-sized pulmonary nodules in a Chinese dedicated cancer hospital. Cancer Biol Med. 2020;17:199-207. doi:10.20892/j.issn.2095-3941.2019.0028
21. Jin X, Zhao SH, Gao J, et al. CT characteristics and pathological implications of early stage (T1N0M0) lung adenocarcinoma with pure ground-glass opacity. Eur Radiol. 2015;25:2532-2540. doi:10.1007/s00330-015-3637-z

22. Chu ZG, Liu MQ, Lv FJ, Li Q, Ouyang Y. Differential diagnosis of solitary pulmonary inflammatory lesions and peripheral lung cancers with contrast-enhanced computed tomography. Clinics (Sao Paulo). 2016;71:555-561. doi:10.6061/clinics/2016(10)01

\section{Publish your work in this journal}

The Journal of Inflammation Research is an international, peerreviewed open-access journal that welcomes laboratory and clinical findings on the molecular basis, cell biology and pharmacology of inflammation including original research, reviews, symposium reports, hypothesis formation and commentaries on: acute/chronic inflammation; mediators of inflammation; cellular processes; molecular mechanisms; pharmacology and novel anti-inflammatory drugs; clinical conditions involving inflammation. The manuscript management system is completely online and includes a very quick and fair peerreview system. Visit http://www.dovepress.com/testimonials.php to read real quotes from published authors.

Submit your manuscript here: https://www.dovepress.com/journal-of-inflammation-research-journal 